\title{
A multistep approach for reticle cleaning
}

\author{
N.B. Koster*, J.C.J. van der Donck, J.K. Stortelder, A.J. de Jong, F.T. Molkenboer \\ TNO, Delft, The Netherlands, * $\underline{\text { norbert.koster@tno.nl }}$
}

\begin{abstract}
The introduction of EUV Lithography for the next node has two major obstacles at the moment; the first is source power and reliability and the second is defect free reticles and damage free cleaning of reticles. We present our results on our investigation for damage free cleaning of EUV reticles with remote plasma cleaning for molecular (carbon) contamination and nanobubbles for particle removal. We believe that a multi step approach is necessary for cleaning of reticles as a single cleaning step will not be sufficient for the efficient removal of molecular as well as particle contamination. Remote plasma seems to be the favorable technique for carbon cleaning and repeated cleaning up to 85 $\mathrm{nm}$ of carbon removal shows no degradation of the reticle material.
\end{abstract}

Keywords: EUVL reticle, particle contamination, molecular contamination, plasma cleaning, nanobubbles cleaning, life time.

\section{INTRODUCTION}

Semiconductor manufacturing is awaiting the introduction of EUVL lithography for the next node. In the closing session at the EUVL symposium of 2011 in Miami three problem areas were clearly identified. The first two are directly related to the lithographic step itself, namely EUV source power and secondly reliability and damage free reticles and cleaning of those reticles. The third problem was identified as the lack or late introduction of actinic inspection equipment, hindering the introduction of EUVL in the semiconductor manufacturing world. In the by the Netherlands government funded research program NanoNext TNO has a sub-project called Nanopatterning in which we investigate the possibilities of reticle cleaning for particles and carbon removal as well as reticle repair. The other partners in the Nanopatterning sub project are investigating related problems like carbon growth model, carbon removal mechanisms, particle behavior and transport, which in the end should give a complete understanding of the contamination mechanisms and the preferred mitigation strategies or cleaning method. The other partners investigating these subjects are ASML, Technical University Eindhoven, Technical University Twente, Holst Centre and FOM institute for plasma physics Rijnhuizen all located in the Netherlands. This paper describes our latest results on particle removal by wet cleaning and carbon removal by remote plasma. The ultimate goal of this project is to find a combination of techniques for the two necessary cleaning steps in such a way that these can be combined in a piece of equipment that is capable of fulfilling the needs of the semiconductor manufacturing industry.

\section{PARTICLE REMOVAL BY WET CLEANING}

\subsection{Introduction}

In the quest for cleaning technology for EUV reticles the focus for particle removal is aimed at mild cleaning mechanisms. Since nanobubbles do not require external mechanical energy it is expected to be an interesting option. Previous reports on cleaning properties of nanobubbles either describe electrolysis ${ }^{[1]}$ or the alcohol/water exchange process $^{[2]}$ for the generation of nanobubbles. Both methods have some severe disadvantages. Electrolysis might damage the Ruthenium cap-layer. The alcohol/water exchange process is difficult to control and making technology for it is extremely difficult. Alternative methods of making nanobubbles are available ${ }^{[3][4]}$. In tone of these methods argon gas is dissolved in the water. Nanobubbles are formed at a slightly elevated temperature. This method is expected to be much easier to handle and the absence of alcohols makes it much more environmentally friendly.

The current paper describes results of the use of argon nanobubbles in cleaning. First, the presence of nanobubbles under the applied conditions is showed. Then, removal of particles from wafer surfaces is investigated. 


\subsection{Experimental}

\section{Samples}

PSL particles $(80 \mathrm{~nm}, 400 \mathrm{~nm}, 900 \mathrm{~nm}$ and $5 \mu \mathrm{m}$, Duke scientific) were diluted in Iso-Propanol. A small volume was spin coated on $100 \mathrm{~mm}$ silicon wafers (TopSil). The particle density was determined by TNO dark field particle scanner the Rapidnano $(80 \mathrm{~nm})^{[4]}$, dark field microscopy $(400 \mathrm{~nm}$ and $900 \mathrm{~nm})$ and bright field microscopy $(5 \mu \mathrm{m})$.

For the $5 \mu \mathrm{m}$ PSL particles the particle density was relatively low (14 particles per image). Therefore, multiple images were taken to acquire enough data for removal calculations. The number of particles in the other samples was sufficiently high for reliable statistics.

\section{Experimental procedure}

Liquids forming argon nanobubbles were prepared by degassing ultra pure water during 1 hour at 1 mbar pressure. The degassed water was saturated with argon gas at higher pressure. Water with two gas conditions were prepared: 1,5 Bar ( 1 hours conditioning) and 2 bar (16 hours conditioning). In order to generate nanobubbles, the liquid was heated to 40 ${ }^{\circ} \mathrm{C}$ prior to exposure.

The formation of nanobubbles by water at 2 bar Argon was studied by AFM. A small amount of water was placed on top of a silicon wafer. In tapping mode the surface was scanned.

The removal of particles was studied by placing a droplet of the nanobubbles forming liquid on the wafer. After an exposure time of 5 or 15 minutes the liquid was removed by blowing it of the wafer by pressurized nitrogen.

Each corner of the wafer was exposed to liquids with different conditions. The four conditions were: ultra pure water without gas / 15 minutes exposure (no nanobubbles) (top left), water with 1,5 bar Argon / 5 minutes exposure (top right), water with 1,5 bar /15 minutes exposure (bottom left) and water with 2 bar Argon /15 minutes exposure (bottom right, see Figure1). Figure 1 shows only three droplets of liquid. The fourth condition ( $2 \mathrm{bar} / 15$ minutes) was carried out in a separate experiment. During the exposure of the wafer with $400 \mathrm{~nm}$ PSL to water $2 \mathrm{bar} / 15$ minutes an experimental error occurred and the results cannot be used. After exposure particle densities were determined and compared with the initial particle density.

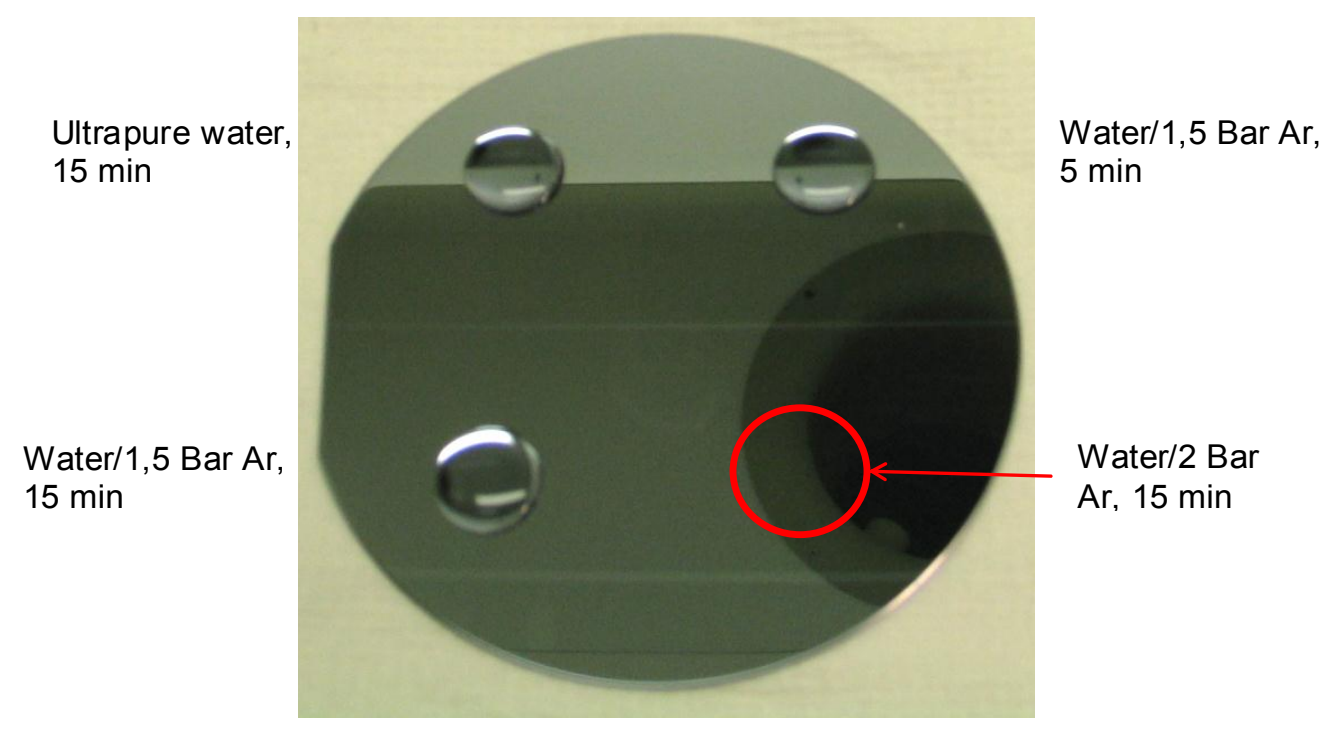

Figure 1. Exposure locations on wafer for liquids with different amounts of nanobubbles.

\subsection{Results}

\section{Nanobubble formation}


Figure 2. shows an AFM image of a surface that is immersed in water with argon ( 2 bar). The white dots show locations with a higher phase shift which proves the presence of nanobubbles. The surface coverage is about $5 \%$.

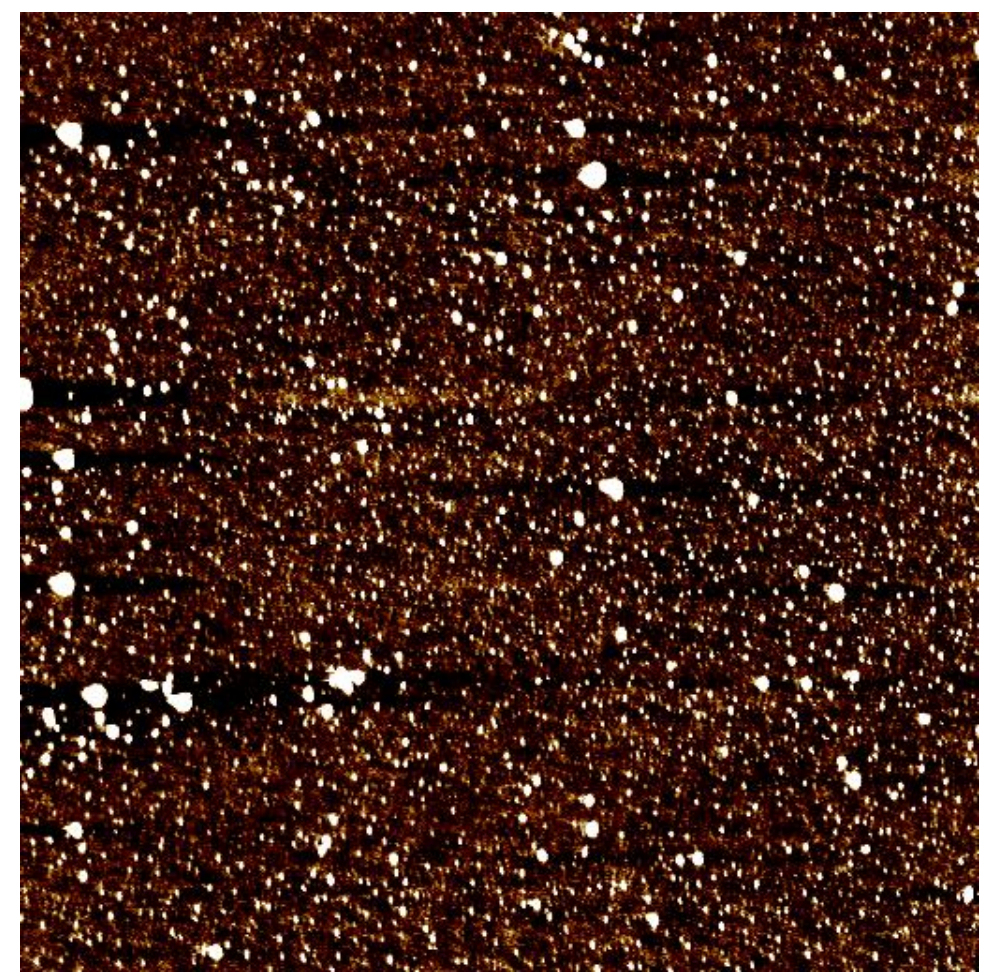

Figure 2. AFM measurement of a surface with nanobubbles. The white dots are the nanobubbles.

\section{Particle removal}

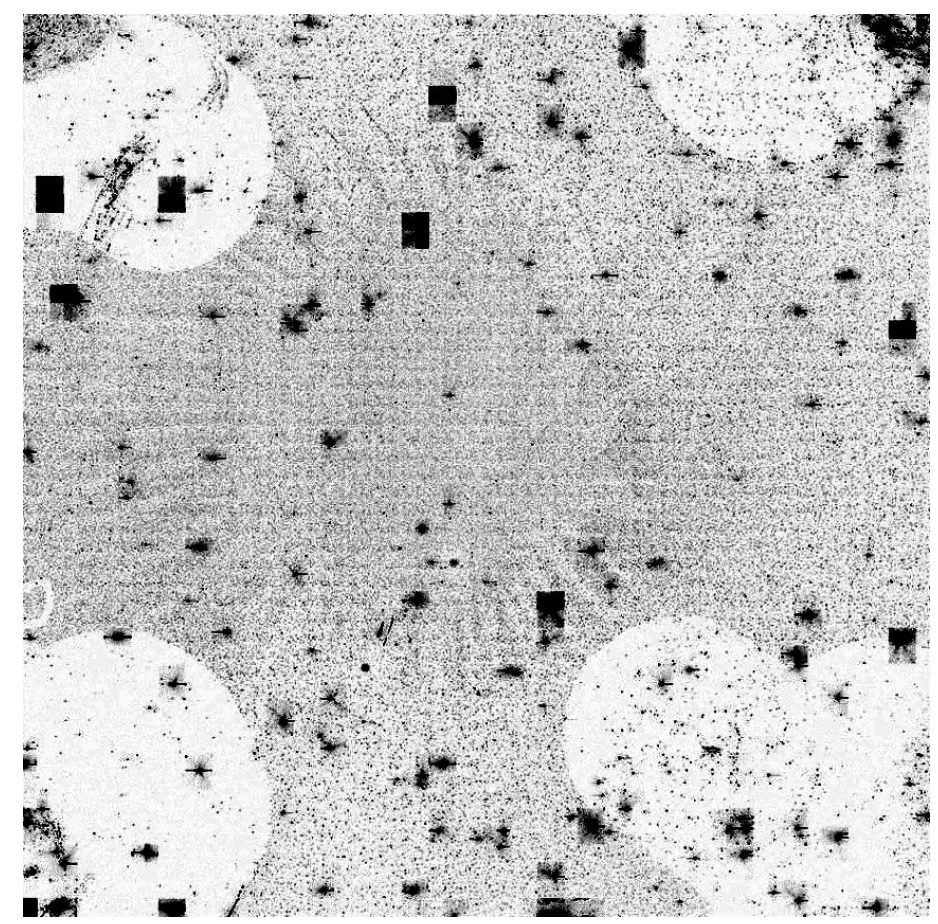

Figure 3. Particles ( $80 \mathrm{~nm}$ PSL) on a wafer determined by Rapidnano ( 70 x $70 \mathrm{~mm}$, negative image). 
Figure 3 shows the particle density on the wafer with $80 \mathrm{~nm}$ PSL particles after exposure. Since the Rapidnano is based on dark field image technology (negative image in this paper) each dark dot represents a particle larger than $60 \mathrm{~nm}$. A light area is present on each corner of Figure 3, where the wafer is exposed to a liquid. Figure 4 gives detail images of the four exposed areas. At a corner of each detail image an unexposed area is present where high numbers of particles are still present. The orientation of the four images is similar to Figure 1. Consequently, the upper left corner was exposed to pure water.

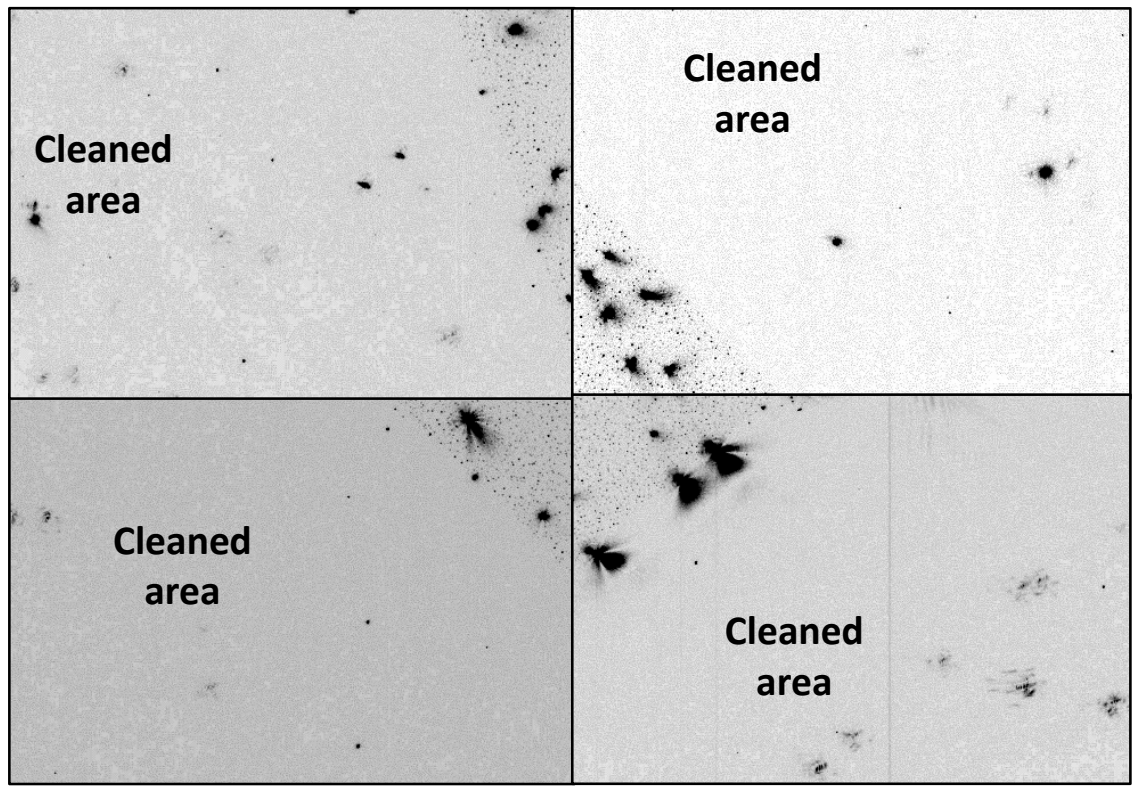

Figure 4. Detail from edges of four black spots ( $80 \mathrm{~nm}$ PSL, negative image, orientation similar to Figure 1).

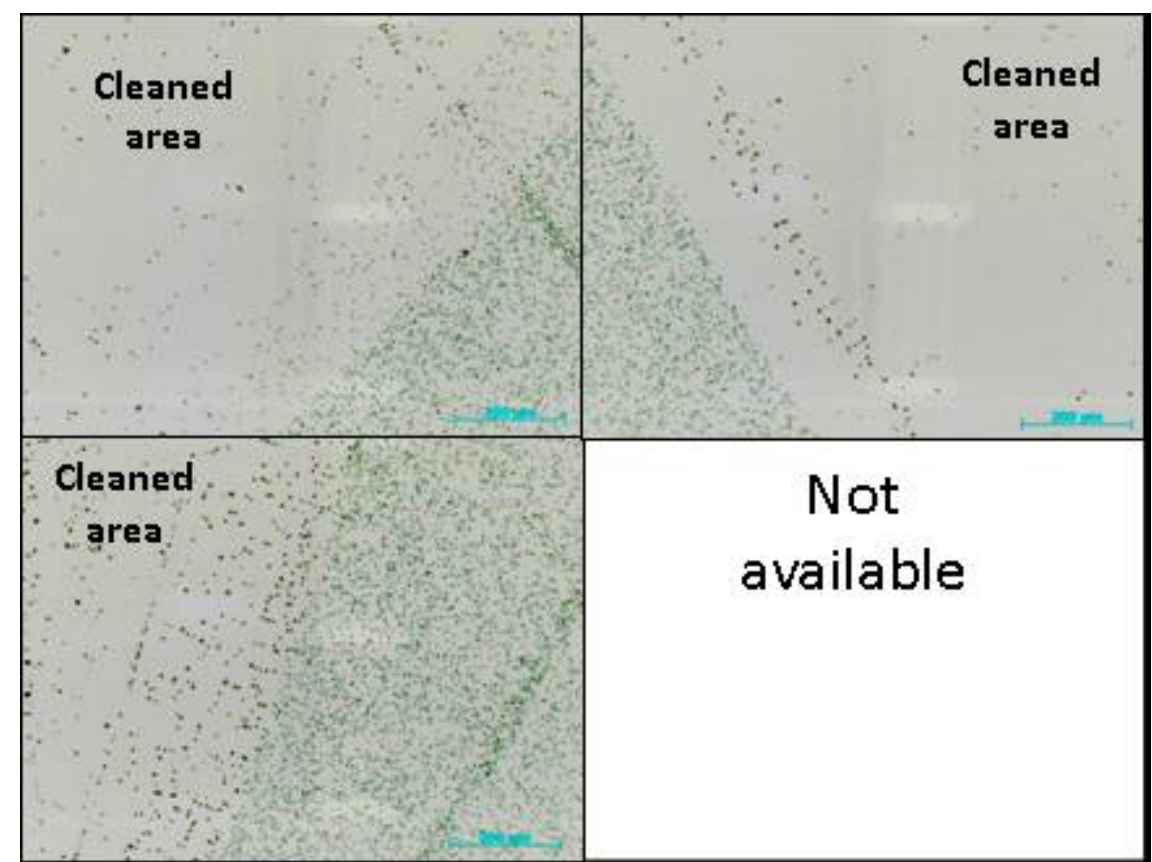

Figure 5. Dark field images (negative) of exposed spots ( $400 \mathrm{~nm}$ PSL, edge), result water $2 \mathrm{bar} / 15$ minutes is omitted because of an experimental error.

Figure 5 shows removal of $400 \mathrm{~nm}$ particles by three liquids. In the images the droplet edge is clearly visible. The unexposed area shows a higher and homogenous particle density than in the exposed area. Close to the edge of the 
exposed area some of remaining the particles are clustered. In the centre of the exposed area the removal rate is higher than at the edge. Because of an experimental error the image of the location for water $2 \mathrm{bar} / 15$ minutes is omitted.

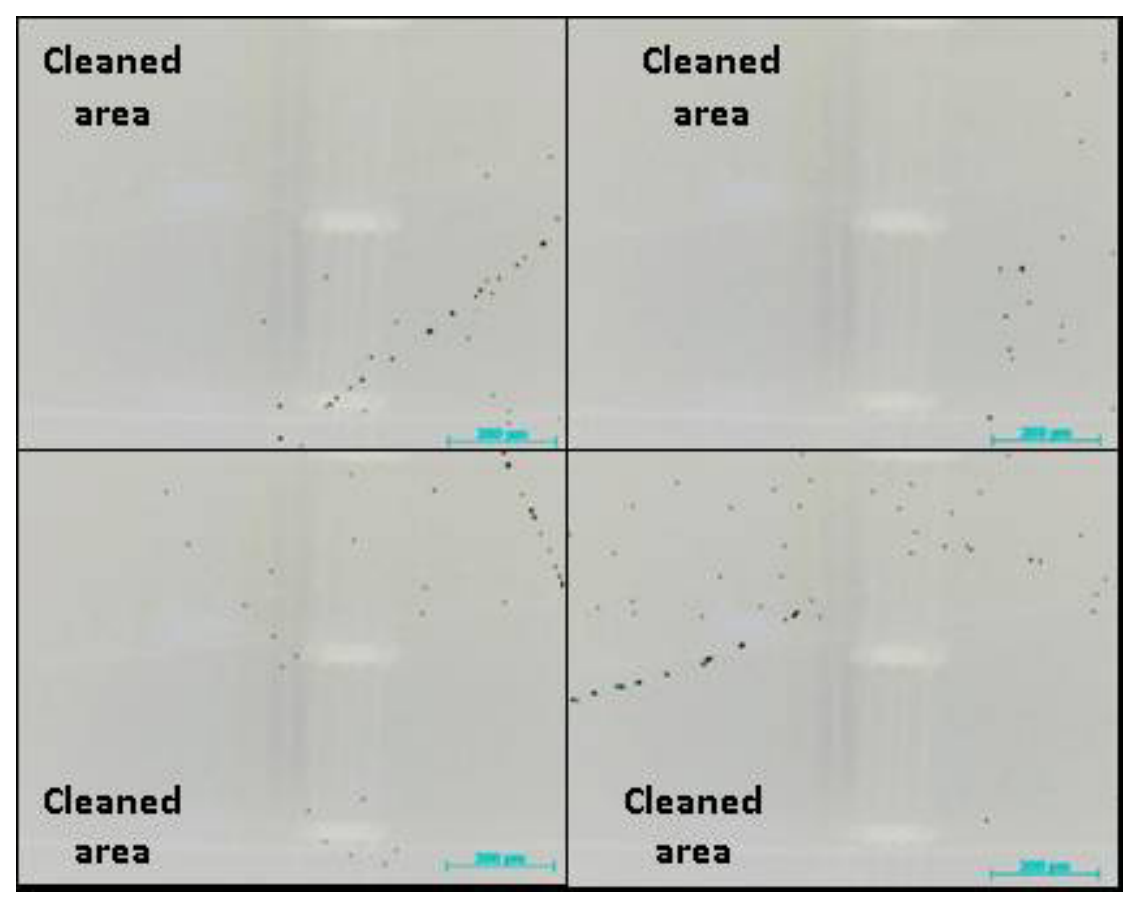

Figure 6. Dark field images (negative) of exposed spots (900 nm, edge).

Figure 6 shows images of the edges of exposed spots on the wafer with $900 \mathrm{~nm}$ PSL particles. At most locations a good removal was observed. Only the location exposed to water 1,5 bar/15 minutes showed a lower removal.

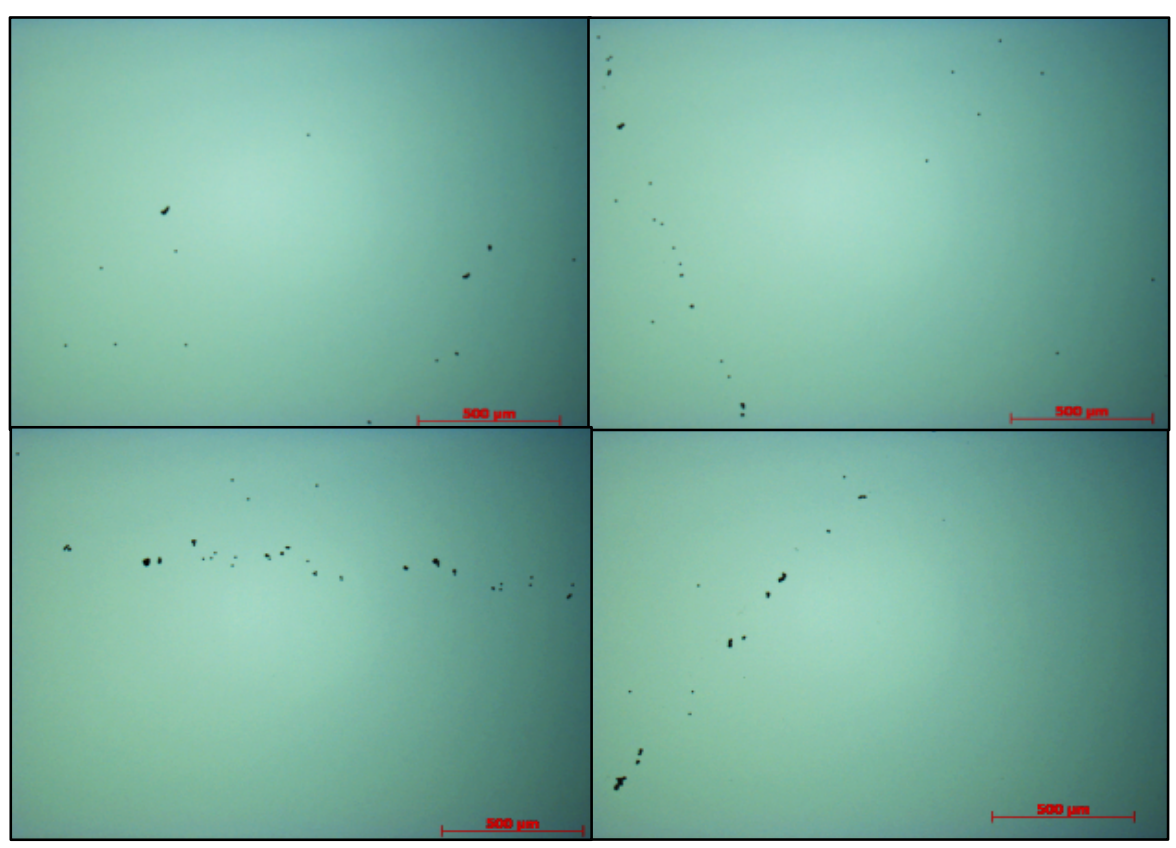

Figure 7. Removal of $5 \mu \mathrm{m}$ particles by liquid

Figure 7 gives microscopy images of the four locations on the wafer with $5 \mu \mathrm{m}$ particles. Overall removal data were estimated for the images (see Table 1). For the $5 \mu \mathrm{m}$ particles 4 to 5 images were used for the evaluation. For the $400 \mathrm{~nm}$ 
and the $900 \mathrm{~nm}$ particles 2 images per location were sufficient for determination of the removal efficiency. With this approach a rough estimate ( $\pm 10 \%$ accuracy) can be made which is sufficient at this stage.

Table 1. Particle removal efficiency of the four different exposure conditions

\begin{tabular}{|l|l|l|l|l|}
\hline & $\mathbf{8 0} \mathbf{n m}$ & $\mathbf{4 0 0} \mathbf{n m}$ & $\mathbf{9 0 0} \mathbf{n m}$ & $\mathbf{5} \boldsymbol{\mu m}$ \\
\hline $\begin{array}{l}\text { Water (no } \\
\text { nanobubbles) }\end{array}$ & $95 \%$ & $50 \%$ & $75 \%$ & $75 \%$ \\
\hline 1,5 bar, 5 min. & $95 \%$ & $90 \%$ & $95 \%$ & $20 \%$ \\
\hline 1,5 bar, 15 min. & $95 \%$ & $75 \%$ & $75 \%$ & $90 \%$ \\
\hline 2 bar, 15 min. & $95 \%$ & Exp. error & $75 \%$ & $75 \%$ \\
\hline
\end{tabular}

From Table 1 it can be concluded that all liquids showed a high particle removal efficiency. No clear correlation between removal efficiency and particle size or removal efficiency and type of liquid could be established. It appears that in the current set-up another removal mechanism was dominant over the removal by nanobubbles. From these experiments no evidence for the contribution of nanobubbles to particle removal was obtained.

\section{CARBON REMOVAL BY REMOTE PLASMA}

\subsection{General}

In our previous investigations ${ }^{[6][7]}$ we have shown that our Shielded Microwave Induced Remote Plasma (SMIRP) is capable of cleaning Multilayer Mirrors (MLM) without damage and that we also can remove organic particles (PSL spheres) in patterned structures. Our latest research focused on further investigating the possibilities of thick carbon layer removal and damage to patterned structures. For this we build a small setup to grow hydrogenated carbon layers by ebeam illumination on several substrates and we deposited TaN layers on substrates for patterning with a thickness representative for EUV reticle absorber layers.

\subsection{Carbon growth set up}

The carbon growth setup was constructed from a DN100CF 6 way cross on a Pfeiffer turbomolecular pump set with a pump speed of 260 1/s. For the illumination we used a Kimball Physics e-gun model which we operated at $1 \mathrm{kV}$ acceleration voltage and $300 \mu \mathrm{A}$ beam current. The base pressure of this system after a night of pumping and without bake out is below $1 * 10^{-6} \mathrm{~Pa}$, when we bake the system for conditioning the base pressure is below $5^{*} 10^{-8} \mathrm{~Pa}$. This ensures that the partial pressure of $\mathrm{O}_{2}$ is very low (below $1^{*} 10^{-7} \mathrm{~Pa}$ ) as this might hinder the growth of carbon due to oxidation of the carbon layer by oxygen ions. These ions are formed by the collision of the oxygen molecules with the primary e-beam or by the secondary ions emitted from the surface of the sample. It is known from literature ${ }^{[8]}$ that introducing oxygen into a system with energetic photons or electrons reduces the carbon growth on a surface.

As model contaminant we used dodecane as this has a relatively high vapor pressure ensuring a stable pressure in the chamber during illumination. After exposure to electrons the remaining dodecane is easily removed by the pumping system and a background check with a Residual Gas Analyzer (RGA) is performed to check for leaks or other unexpected gas species. The dodecane pressure during illumination is $1 * 10^{-4} \mathrm{~Pa}$ and in combination with the low base pressure of our system this ensures that we have a $100 \%$ dodecane gas environment and no residual oxygen that limits the growth rate of carbon. Using these setting we are able to grow $9 \mathrm{~nm} /$ hour as can be seen in figure 8 .

The spot size is roughly $1.5 \mathrm{~mm}$ in diameter and shows a central peak about twice the height of the average height. We believe that this is caused by astigmatism in the e-beam and the used deflection setting of the e-gun but this was the best focus we could obtain and provides a good carbon spot on the samples. 

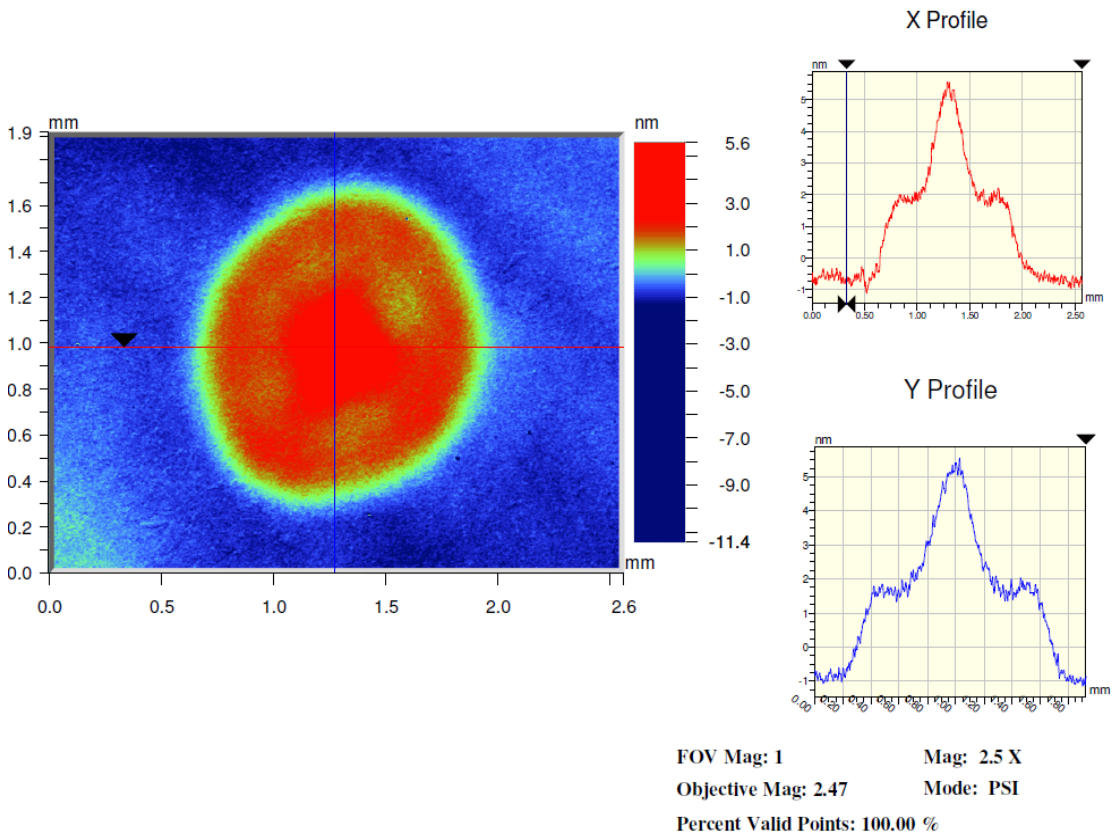

Figure 8. Interferometer data of grown carbon spot

\subsection{Carbon cleaning of multilayer}

To test the carbon growth and removal we exposed a 1" multilayer mirror in the growth set up for 45 min, leaving a spot with an approximate height of $6.6 \mathrm{~nm}$ of carbon. The mirror was 4 years old, but XPS analysis showed no degradation of the mirror. This was not confirmed by EUV reflectometry, a reference sample was send to the PTB also but no data is available of the reference sample at the moment of writing this paper. The sample was then transferred to our plasma cleaner and repeatedly cleaned for 10 minutes. The settings used for the plasma were: $1000 \mathrm{~W}$ microwave power, $50 \%$ duty cycle, $215 \mathrm{sccm} \mathrm{H}_{2}$ and a pressure of $56 \mathrm{~Pa}$. After each cleaning step of $10 \mathrm{~min}$ the chamber was vented and by optical inspection it was verified if the spot was still present. After 8 cleaning cycles the spot was not visible by eye anymore and we measured the spot with the interferometer. At the tenth cleaning cycle the spot was below $1 \mathrm{~nm}$ in height but still clearly visible with the interferometer, see figure 9 for the surface map.
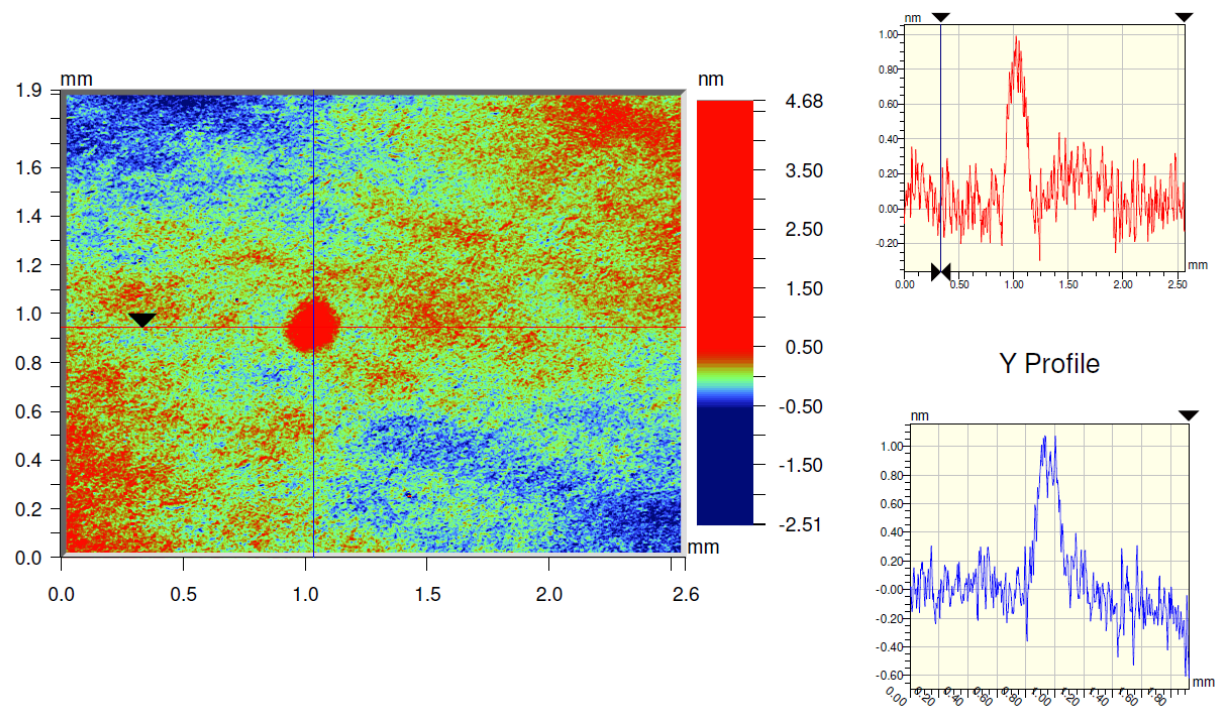

Figure 9. Interferometer data of carbon spot after 10 cleaning cycles 
With a total of 12 completed cleaning cycles the spot is not visible by interferometer anymore and the sample was transferred to the XPS for surface analysis. The XPS data showed a little carbon, about $0,5 \mathrm{~nm}$ thick, which was uniform over the whole sample. This is most likely caused by the atmospheric transfer of the sample to the XPS. The analysis showed that the caplayer was intact, the analysis also showed presence of $\mathrm{Sn}, \mathrm{Zn}$ and $\mathrm{Pb}$ outside the spot. A witness sample showed the presence of the same elements. After a mechanical abrasive cleaning of the chamber the numbers of $\mathrm{Sn}, \mathrm{Zn}$ and $\mathrm{Pb}$ were almost below detection limit of the XPS on a witness sample with a Ru coating. This shows that the observed $\mathrm{Sn}, \mathrm{Zn}$ and $\mathrm{Pb}$ are most probably due to cross contamination from the plasma chamber. The sample was the send to the PTB in Berlin for EUV reflectometry. As can be seen from the surface map in figure 10 the spot at location $\mathrm{x}=3, \mathrm{y}=6$ is clearly visible as a region of higher reflectivity. The losses caused by the redeposition and possibly aging of the mirror attributed to a relative reflectivity loss of $6 \%$ Also there is a gradually decrease in reflectivity visible towards the outside of the sample. At this moment we have no explanation for this effect, we suspect that the increased reflectivity at the location of the original carbon spot is caused by the masking effect of the carbon during cleaning, which prevented that the contaminants from the chamber are deposited at that location. In effect the carbon acts as a prevention layer for redepostion of contaminants.
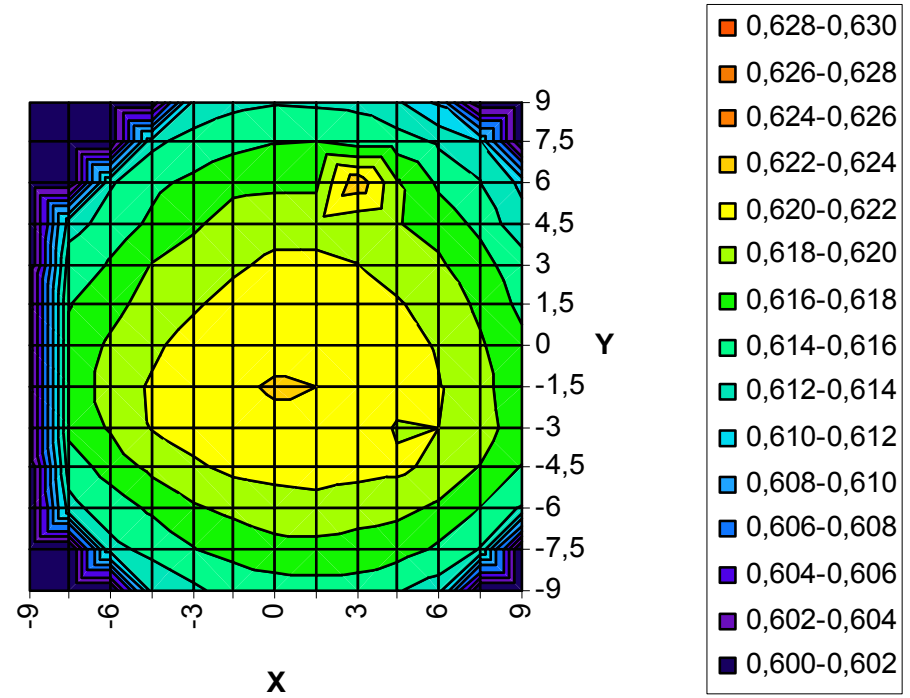

Figure 10. EUV reflectometry data of carbon spot after 10 cleaning cycles

\subsection{Carbon removal from TaN absorber and trenches}

To further investigate the possibility of remote plasma cleaning we sputter deposited $80 \mathrm{~nm}$ of TaN on a glass substrate and cleaned the surface for 50 min with intervals of $10 \mathrm{~min}$ and imaged with SEM and analyzed the surface with EDS. In figure 11 the EDS spectrum is shown before and after cleaning.

The spectra reveal that the TaN layer has not changed in composition, even after $50 \mathrm{~min}$ of cleaning. In the spectra after cleaning a carbon peak is still visible, but this has reduced significantly. As we know that the SEM used for the elemental analysis is polluted the carbon can also originate from the measurement itself.

To further study the effect of plasma cleaning on trenches we deposited $400 \mathrm{~nm}$ of TaN on silicon and used the Helium Ion Microscope (HIM) ${ }^{[9]}$ to etch trenches in the TaN. The etching was performed with $\mathrm{XeF}_{2}$ as precursor gas and with different exposure conditions resulting with more undercut in some of the 4 structures as can be seen in Figure 12. The structure had a line width of $80 \mathrm{~nm}$ with a pitch of $300 \mathrm{~nm}$. We used the SEM to grow the carbon instead of our carbon growth setup because it was not possible to find the location of the structure without the added assistance of the SEM. A carbon layer was deposited by exposing the layer for $7 \mathrm{~min}$ in the SEM and subsequently cleaned with plasma for 20 min. After this cleaning no carbon was visible with the SEM. A second layer of carbon was deposited in by the SEM on the same location by exposing for another $7 \mathrm{~min}$ with the SEM. This layer was again cleaned for 20 min with the $\mathrm{H}_{2}$ plasma and imaged. The image showed no visible remaining carbon on the TaN absorber layer, also the trenches appear to have the same shape, size and undercut as before the contamination and cleaning had been executed. We intend to investigate this in more detail in the remaining time of the project. 


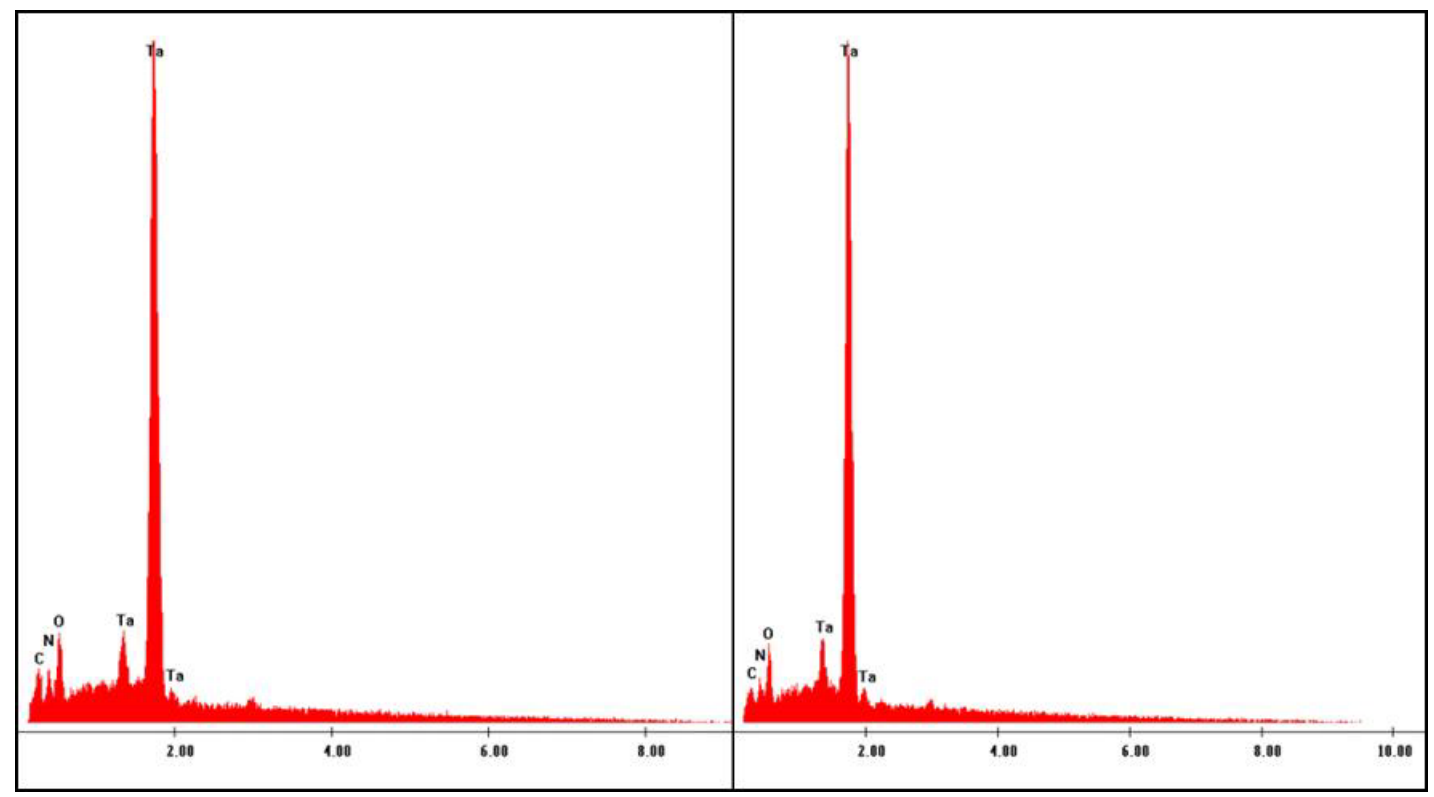

Figure 11. EDS spectra of TaN layer, left with carbon contamination, right after 50 min of plasma cleaning

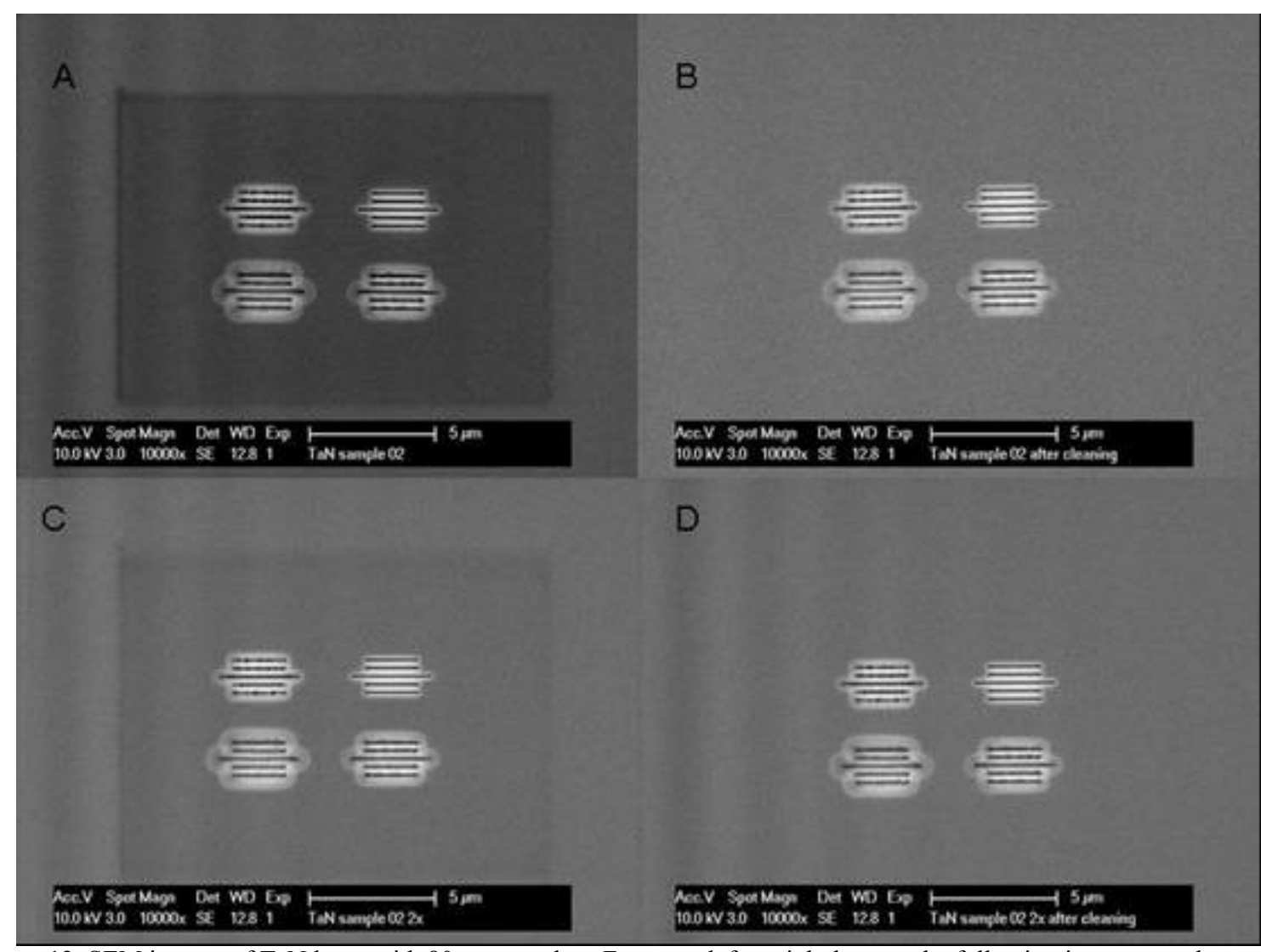

Figure 12. SEM images of TaN layer with $80 \mathrm{~nm}$ trenches. From top left to right bottom the following images are shown a) image after 7 min exposure with SEM b) after 20 min plasma cleaning c) again 7 min of exposure with SEM and d) after a second 20 min of plasma cleaning. The carbon contamination is visible as a square spot. 
The images displayed in figure 12 show no sign of degradation of the TaN layer or changes in the pattern. A more detailed analysis of the layer composition with EDS shows that after 40 min of cleaning in the plasma chamber no redeposition of the earlier observed elements $\mathrm{Sn}, \mathrm{Zn}$ and $\mathrm{Pb}$ has occurred. This indicates that the performed mechanical abrasive cleaning on the chamber was indeed successful. The EDS spectrum is shown in figure 13.

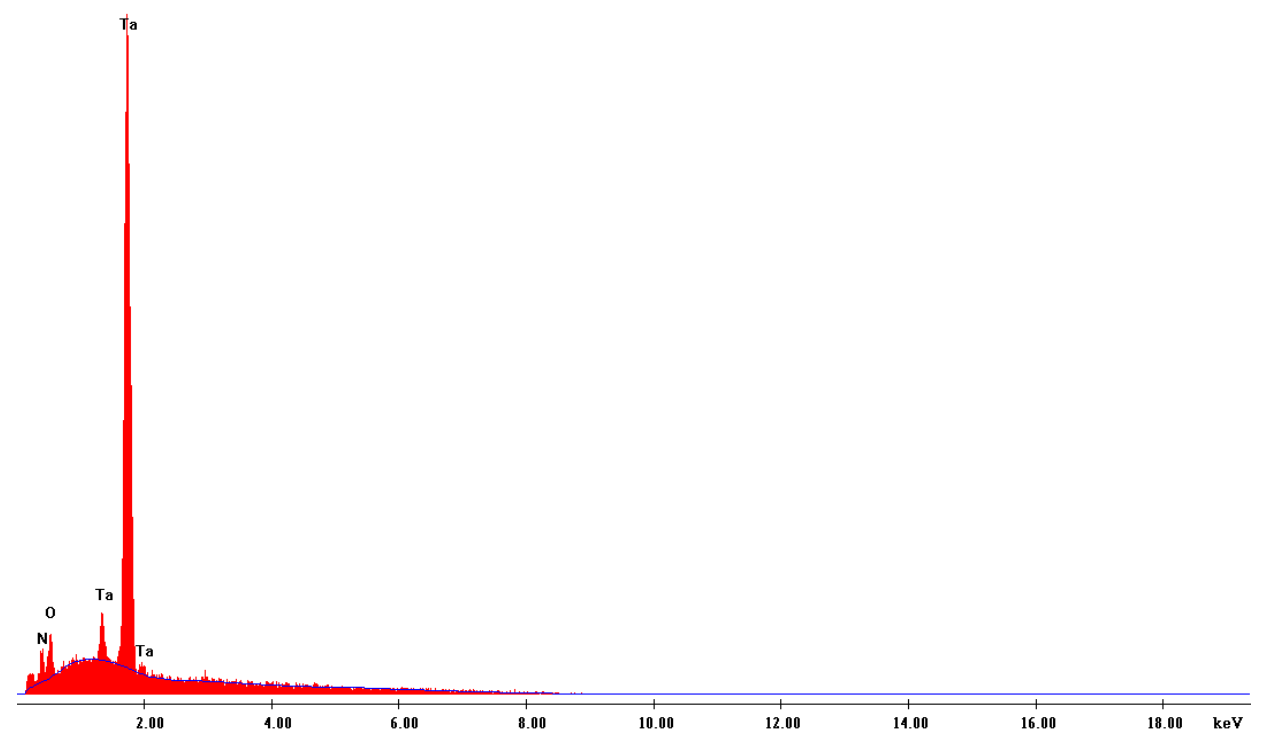

Figure 13. SEDS spectra of TaN layer after $40 \mathrm{~min}$ of total plasma cleaning

\section{DISCUSSION}

The first experiments with the Argon/water method have shown the existence of nanobubbles. The removal rate by a different mechanism was higher than a possible contribution by the nanobubbles. Therefore, no conclusive effect of increased particle removal by nanobubbles has been observed. We intend do more research on this topic and more thoroughly investigate the formation of nanobubbles and their possibility for particle removal. This includes a feasibility study for scaling the process to industrial sizes of substrates.

The removal of carbon by remote plasma has been shown and no damage to substrates has been observed, except for the redeposition due to a contaminated chamber. For the industrial application of plasma this is a point of concern and care should be taken that such a system does not get contaminated by unapproved usage.

\section{ACKNOWLEDGEMENTS}

We would like to thank J.H. van den Berg for the SEM images, EDS analysis and TaN deposition, H.H.P.Th. Bekman for the interferometer measurements and E. van Veldhoven and D.J. Maas for the HIM etching of the structures, all from TNO. We also would like tot thank R. Berkelaar from Technical University Twente for doing the nanobubble experiments. We also would like to thank Carl Zeiss SMT for providing beam time at PTB for reflectometry measurements and samples. This work was made possible by funding by the Dutch government within the NanoNext program.

\section{REFERENCES}

[1] Zhinhua Wu, Hongbing Chen, Yaming Dong, Huiling Mao, Jielin Sun, Shenfu Chen, Vincent S.J. Craigh and Jun $\mathrm{Hu}$, "Cleaning using nanobubbles: Defouling by electrochemical generation of bubbles", J. Colloid and Interface Sci., 328 10-14 (2008) 
[2] Shangjiong Yang, Anton Duisterwinkel, Jetske Stortelder and Jacques van der Donck, "Particle cleaning of EUV reticles", EUVL symposium, Miami, 2011

[3] Seddon, J. R. T., Harold J.W. Zandvliet, and Detlef Lohse, "Knudsen Gas Provides Nanobubble Stability", Phys. Rev. Lett., 107, 116101 (2011)

[4] Seddon J R T, Kooij E S, Poelsema B, Zandvliet H J W and Lohse D "Surface bubble nucleation phase space", Phys. Rev. Lett., 106, 056101 (2011)

[5] van der Donck, J, Snel, R., Stortelder J,. Abutan, A, Oostrom, S, van Reek, S, van der Zwan, B, van der Walle, P.,"Particle detection on flat surfaces", Proc. of SPIE Vol. 7969, 79691S, (2011)

[6] Koster, N.B. et al, "New developments in cleaning of EUVL mirrors and reticles", EUVL symposium2009, Praque, http://www.sematech.org/meetings/archives/litho/8653/pres/O_RC-03 Koster_TNO.pdf, (2009)

[7] Koster, N.B. et al, "Towards defect free reticles: carbon and particle removal by a single dry cleaning process and pattern repair by HIM", SPIE proceedings Advanced lithography symposium 2011, VOL 7969-31, (2011)

[8] Katsuhiko Murakami et al, "Development of EUV tool technologies in Nikon", EUVL symposium 2011, Miami, (2011)

[9] Maas, D.J. et al, "Nano engineering with a focused ion beam", MRS spring meeting 2011, MRS PROC.1354 MRSS11-1354-II03-03, (2011) 